\title{
Tasa de crecimiento de espigas de trigo (Triticum aestivum) en diferentes niveles de radiacion incidente en Corrientes
}

\author{
Balbi'1, Celsa N.; Rodríguez, Erica, Neiff, Nicolás \\ 'Campo Experimental -FCA UNNE - cnbalbi@agr.unne.edu.ar
}

\begin{abstract}
Resumen
El trigo (Triticum aestivum) es el cultivo invernal más importante de Argentina y en la región NEA es utilizado en la rotación. En condiciones potenciales, el crecimiento del cultivo depende de la radiación solar y de la temperatura, que se vinculan en el cociente fototermal (Q). En el intervalo desde 20 días prefloración a 10 días posfloración se genera el número de granos (NG), el cual está ligado al crecimiento de espigas (CE) durante este período. El objetivo de este trabajo fue determinar el efecto de la radiación solar en el período de crecimiento de espigas de trigo y relacionarlo con el rendimiento potencial. Se instalaron experimentos en el Campo Experimental de la Facultad de Ciencias Agrarias (UNNE), Corrientes, durante los años 2009 y 2010. Se sembró la variedad Baguette 13 el 29 de mayo y el 2 de junio respectivamente, sin limitantes hídricas ni nutricionales, y se ensayaron tres niveles de radiación aplicados durante el período de crecimiento de espigas (testigo, S50\% y S80\%). Se determinó el rendimiento, sus componentes numéricos (NG y PG), CE y la eficiencia del uso de la radiación (EUR). Los rendimientos promedio de ambas campañas oscilaron entre 6895,55 y 3402,10 Kg ha-1 $(\mathrm{p}=0,0002)$; mientras que el NG osciló entre 17245,91 y 9267,36 ( $\mathrm{p}=0.0004)$. En el PG, el testigo $(40,00 \mathrm{~g})$ se diferenció de los tratamientos $\mathrm{S} 50 \%$ y S80\% ( $\mathrm{p}=0.0930)$, no encontrándose diferencias entre los dos últimos (37.63 y $37.90 \mathrm{~g}$ respectivamente). Se ajustaron tres ecuaciones diferentes para el CE. Se encontraron asociaciones entre rendimiento y $\mathrm{NG}\left(\mathrm{R}^{2}=0.98\right)$, y éste último se asoció positivamente $(\mathrm{r}=0.62)$ con el peso de espigas al finalizar el período de crecimiento de las mismas.
\end{abstract}

Palabras clave: trigo, crecimiento de espigas, radiación.

\begin{abstract}
Summary
Wheat (Triticum aestivum) is the most important winter crop in Argentina. In the North East region, it is commonly used in rotation. Potential growth depends on solar radiation and temperature, combined in the photothermal quotient (Q). The grain number (GN) is determined in the interval from 20 days before to 10 days after anthesis, and this is related to spike growth (SG) during this period. The aim of this study was to determine the effect of solar radiation on the spike growth period of wheat, and to relate it to yield potential. Two experiments were performed in the experimental field of Facultad de Ciencias Agrarias (UNNE), Corrientes, during the growing seasons of 2009 and 2010, under potential conditions (i.e., without water and nutrient limitations). Baguette 13 variety was planted on May 29 and June 2, and three radiation levels were tested during the spike growth period (control, S50\% and S80\%). Yield, its numerical components (GN and GW), SG and radiation use efficiency (RUE) were determined. Average yields of both years ranged from 6895.55 to $3402.10 \mathrm{~kg} \mathrm{ha}^{-1}$ ( $\left.\mathrm{p}=0.0002\right)$, NG varied between 17245.91 and $9267.36(\mathrm{p}=0.0004)$ and, for $\mathrm{GW}$, the control $(40.00 \mathrm{~g})$ differed from the $\mathrm{S} 50$ and the S80 treatments $(\mathrm{p}=0.0930)$, with no differences between the last two (37.63 and $37.90 \mathrm{~g}$, respectively). Three different equations were fitted to $\mathrm{SG}$. Associations were found between yield and GN $\left(\mathrm{R}^{2}=0.98\right)$, and the last one was positively associated $(\mathrm{r}=0.62)$ with spike weight at the end of the spike growth period.
\end{abstract}

Key words: wheat, spikes growth, radiation.

\section{INTRODUCCIÓN}

El trigo pertenece a la familia de las gramíneas (Poaceae), siendo las variedades más cultivadas T.durum y T. compactum. El trigo harinero hexaploide llamado T. aestivum es el cereal panificable más cultivado en el mundo (Satorre et al., 2003). Es uno de los principales cultivos de la pampa húmeda y quizás el grano más antiguo cultivado en la Argentina. $\mathrm{Su}$ producción ha evolucionado de manera positiva desde mediados de los' 90 apoyada en los altos rendimientos y en la ampliación de la superficie destinada a este cereal que ha incorporado nuevas áreas en la zona norte del país. En la actualidad, Argentina produce unos 15 millones de toneladas por año. Como el volumen de uso interno del trigo (alrededor de 5 millones de tn) se mantiene estable, tal evolución ha incrementado significativamente el excedente exportable, por lo cual nuestro país se ubica entre los 
cinco mayores exportadores de trigo del mundo con una participación que ronda el $10 \%$ del total mundial (Seghezzo y Molfese, 2006).

En condiciones de rendimiento potencial (sin limitantes de agua, nutrientes y libre de adversidades) el crecimiento del cultivo de la cantidad de radiación solar que intercepta y de la temperatura. La cantidad de radiación fotosintéticamente activa que capta un cultivo depende de la cantidad de radiación que incide sobre el suelo y de la cobertura que éste logra. El otro factor climático que influye sobre el rendimiento potencial es la temperatura, la que actúa acortando la duración de las etapas. Se ha definido un índice, que relaciona el efecto positivo de la radiación y el negativo de la temperatura sobre el número de granos. Este índice se denomina cociente fototermal (Q) y se relaciona directamente con el número de granos (Fischer, 1985).

En trigo, el número de granos queda definido a través de las distintas etapas del cultivo $y$, fundamentalmente, dentro de la etapa reproductiva. Sin embargo, no todas esas etapas tienen igual importancia relativa en el logro del número de granos por $\mathrm{m}^{2}$, ya que existe un período crítico para la definición de dicho componente. El lapso que media desde 20 días prefloración y 10 posfloración, es de suma importancia para la generación del número de granos por unidad de área (Fischer, 1985) y esto está ligado a la tasa de crecimiento de espigas durante este período (Abbate et al., 1997a). Las espigas empiezan a crecer cuando tienen solo el 5\% de su peso final, esto ocurre $20-25$ días antes de antesis. Entonces, el período de crecimiento de las espigas dura 27-32 días dependiendo de las condiciones ambientales, con poca variación entre cultivares (Abbate, 2004). El lapso desde 20 días prefloración y 7 a 10 posfloración, es de suma importancia para la generación de números de granos por unidad de área y esto está ligado a la tasa de crecimiento de espigas durante este período (Abbate et al., 1997a).

Así, las condiciones ambientales que se presentan durante este período crítico, inciden de manera importante en el número final de granos establecidos. Durante dicho período se define el número de macollos que finalmente llegan a producir espigas, estableciendo el número de espigas por unidad de área y el número de primordios florales que sobrevivan en cada una de las espiguillas, obteniéndose, como resultado final, el número de flores fértiles a floración, las que finalmente luego del cuaje, quedan establecidas como grano. Del producto de ambos componentes (número de espigas. $\mathrm{m}^{-2}$ y granos.espiga ${ }^{-1}$ ) surge el número de granos por unidad de área.

La relación positiva entre el peso de las espigas y el número de flores fértiles en floración, demuestra que la cantidad de recursos alojados en la espiga es fundamental para lograr un alto número de granos (Slafer et al., 1990). De esta manera ante valores iguales de partición de asimilados a espigas, una mayor cantidad de biomasa acumulada a floración determina un mayor peso de las espigas y por lo tanto un mayor número de granos. Siendo que la radiación solar afecta al número de granos a través de su relación lineal positiva con la tasa de crecimiento del cultivo, mientras que la temperatura lo afecta negativamente, acelerando el desarrollo, es posible pensar que el número de granos (y en muchos casos el rendimiento) depende de estos dos factores ambientales durante el periodo crítico definido alrededor de la antesis (Miralles, 2003).

En este sentido se desconoce el efecto de la radiación incidente en la tasa de crecimiento de espigas de trigo durante el período crítico en el noreste argentino. Cuantificar las relaciones entre las variables ambientales y los componentes de rendimiento permitiría optimizar las prácticas de manejo tendientes a maximizar los rendimientos y la calidad.

\section{OBJETIVO}

El objetivo de este trabajo fue determinar el efecto de la radiación solar interceptada durante el período crítico del trigo sobre la tasa de crecimiento de espigas, y relacionarlo con su rendimiento potencial.

\section{MATERIALES Y MÉTODOS}

\section{Material biológico - características principales de la variedad}

Se sembró la variedad de trigo Baguette Premium 13 perteneciente a la empresa Nidera semillas, de ciclo vegetativo intermedio corto, porte vegetativo semi erecto, capacidad de macollaje media, comportamiento a vuelco excelente y caña fuerte.

\section{Lugar de Realización y tipo de suelo y clima:}

Los experimentos se realizaron en el Campo Experimental de la Facultad de Ciencias Agrarias de la UNNE, localidad de Corrientes, durante los ciclos agrícolas 2009 y 2010.

El Campo Experimental está ubicado en las siguientes coordenadas geográficas: latitud: $27^{\circ}$ 28'27.23"S; longitud: 58 47'00.66” O; y altitud: 50 $\mathrm{m}$ sobre el nivel del mar. El suelo está clasificado como Udipsament álfico, mixto, hipertérmico perteneciente a la serie Ensenada Grande (Escobar et al., 1994). El clima de la región está clasificado como clima templado húmedo según Köppen (De Fina y Ravelo, 1985; Strahler y Strahler, 1997; Pascale y Damario, 2004).

\section{Manejo del Cultivo:}

El lote se preparó 30 días antes de la siembra. Los días 29 de mayo de 2009 y 2 de junio de 2010 se 
sembró la variedad Baguette Premium 13, manualmente a chorrillo continuo. En ambas campañas se aplicó herbicida metsulfurón en preemergencia con una dosis de 10 g.ha $^{-1}$. Se realizaron aplicaciones de herbicidas e insecticidas según la necesidad de controlar malezas y plagas.

\section{Tratamientos:}

Se realizaron tratamientos tendientes a reducir la radiación solar incidente, consistente en sombreos aplicados desde 20 días antes hasta 7 días posteriores a floración. Se aplicaron tres tratamientos: Testigo: sin sombreo, S50: con malla media sombra comercial de $50 \%$ y S80: malla media sombra comercial de $80 \%$. La radiación incidente real medida durante el ensayo en cada uno de los tratamientos con sombreo se puede observar en la Tabla 1.

Los porcentajes reales de sombreo logrados con la malla media sombra fueron $46.30 \%$ y $70.07 \%$ para los tratamientos S50\% y S80\% respectivamente. En base al porcentaje real se obtuvieron todos los resultados, sin embargo se denominó a los tratamientos S50 y S80 durante el texto del trabajo.

El período sombreado fue 27 días para el año 2009 y 29 días para 2010. La radiación fotosintéticamente activa (PAR) acumulada durante ese período fue en promedio de $162,3 \mathrm{MJ} \mathrm{m}^{-2}$ para el testigo, 87,51 MJ $\mathrm{m}^{-2}$ para $\mathrm{S} 50$ y $50,69 \mathrm{MJ} \mathrm{m}^{-2}$ para $\mathrm{S} 80$.

El período sombreado coincidió con el período crítico citado por Abbate et al. (1997b). Los ensayos fueron conducidos en condiciones potenciales, sin limitantes hídricas ni nutricionales, lo que se logró a partir de fertilizaciones y riego por goteo, con lo que eliminan la posibilidades de estreses abióticos.

\section{Mediciones: \\ Radiación}

Los datos de radiación solar fueron registrados durante la realización de los experimentos en un Data Logger de 8 canales (Empresa Cavadevices, Buenos Aires, Argentina), compuesto de un sensor de radiación PAR, (celda de GaAsP con respuesta espectral en la banda comprendida entre los 400 y los $700 \mathrm{~nm}$ de longitud de onda) y dos sensores de temperatura (aire y suelo de $-40 \mathrm{a}+125^{\circ} \mathrm{C}$ ). Los estados fenológicos se registraron de acuerdo con la escala adaptada de Slafer y Rawson (Satorre et al., 2003).

La intercepción de PAR por el cultivo se midió utilizando un sensor lineal quantum (Cavadevices, B.A, Argentina). Se realizaron 5 mediciones por parcela ubicadas desde el centro de un surco, de acuerdo a la metodología propuesta por Gallo y Daughtry (1986) en tres momentos: al comienzo y final del período crítico y en floración, por encima y por debajo de la malla de media sombra de acuerdo a los tratamientos aplicados.
Tabla 1. Caracterización de los tratamientos: Valores de radiación incidente reales sobre el canopeo del cultivo en los distintos tratamientos. Testigo (sin sombreo), S50 (sombreo con malla media sombra de 50\%) y S80 (sombreo con malla media sombra de $80 \%$ ).

\begin{tabular}{|c|c|c|}
\hline Tratamiento & Radiación $\left(\mu \mathrm{MOL} \mathrm{m} \mathrm{m}^{-2} \mathrm{~s}^{-1}\right)$ & $\%$ de incidencia \\
\hline Testigo & 1226 & 100 \\
\hline S 50 & 659 & 53,70 \\
\hline S 80 & 367 & 29,93 \\
\hline
\end{tabular}

\section{Biomasa}

La materia seca de planta entera de una superficie de $0.25 \mathrm{~m}^{2}$ se obtuvo en tres momentos: 25 días antes de floración, 10 días después de floración y en madurez fisiológica. En cada momento se particionó en en tallo, hoja e inflorescencia). El material fue secado en estufa a $65^{\circ} \mathrm{C}$ hasta peso constante y posteriormente pesado. Durante el período de crecimiento de la espiga, se midió la tasa de crecimiento a partir de la materia seca de la espiga. Ésta se obtuvo por disección de la planta cada tres o cuatro días, extrayendo la espiga que crecía dentro del tallo. El período crítico o período de crecimiento de espigas, abarcó desde el momento en que la espiga tenía el 5\% de su peso final sin granos hasta la floración (Abbate et al., 1997b).

\section{Rendimiento y sus Componentes}

Cuando los granos tuvieron $18 \%$ de humedad se cosecharon los surcos centrales de cada parcela respetando las borduras de $50 \mathrm{~cm}$. Las espigas se cosecharon manualmente para su posterior trillado en forma mecánica con una trilladora de la Estación Experimental Agropecuaria El Sombrero (INTA Corrientes). Se realizaron los correspondientes análisis a los granos de las distintas parcelas Se determinó rendimiento en grano evaluando peso y número. El peso de los granos se determino a través del conteo de cinco muestras por cada tratamiento y por cada repetición de 500 granos cada una y su posterior pesado con balanza electrónica, (Denver XP300 (3000.1 gr) y Ohaus CS2000 (2000-1 gr)). Para el número de granos se determinó primero el número de espigas por metro cuadrado en cada parcela, contando las espigas que había en un metro lineal por seis líneos de ancho y ajustando el dato a $1 \mathrm{~m}^{2}$. Posteriormente se determinó el número de granos por espiga, obteniendo el número de granos por metro cuadrado.

\section{Diseño Experimental y Análisis Estadístico:}

Los tratamientos se distribuyeron según un diseño de bloques completos al azar en parcelas divididas con cuatro repeticiones cada una, en parcelas de 8 líneas a $0,17 \mathrm{~m}$ por diez metros de longitud, destinando tres metros para cada nivel de sombreo. Los datos se analizaron con análisis de varianza (ANOVA) y test de comparación de medias. Los ajustes de ecuaciones 
se realizaron con Excel. En los casos que se encontraron asociaciones se realizaron las correspondientes

\section{RESULTADOS Y DISCUSIÓN}

\section{Rendimiento y sus componentes}

La Tabla 2 muestra los componentes numéricos del rendimiento en la variedad de trigo Baguette Premium 13 para los tres tratamientos de niveles de radiación ensayados: testigo (sin sombreo), S50 y S80. Los datos son el promedio de dos años de ensayos conducidos sin limitaciones hídricas ni nutricionales.

El rendimiento en grano varió de 6895.55 a 3402.10 $\mathrm{kg} \mathrm{ha}^{-1}(\mathrm{p}=0.0002)$ en los diferentes tratamientos (Tabla 2) observándose diferencias significativas entre ellos. También se encontraron diferencias significativas en lo que respecta al peso de 1000 granos, siendo el Testigo (40.00 g) estadísticamente diferente de los tratamientos S50 y S80 ( $p=0.0930)$. No se observaron diferencias significativas entre estos dos últimos (37.63 y 37.90 g respectivamente). En cuanto al número de granos. $\mathrm{m}^{-2}$ se han encontrado diferencias significativas entre los 3 tratamientos $(\mathrm{p}=0.0004)$.

En lo que respecta al número de espigas por metro cuadrado se encontraron diferencias significativas regresiones.

entre los tratamientos testigo y S50 con respecto al tratamiento $\mathrm{S} 80(\mathrm{p}=0.0391)$ siendo este último más bajo en número de espigas por metro cuadrado. Esto puede atribuirse al nivel de sombreo que ocasionó la infertilidad de macollos, mientras que en el testigo fueron fértiles, o sea que produjeron espigas.

\section{Crecimiento y Peso seco de espigas}

En la Tabla 3 se muestra el peso seco de las espigas registrados en los distintos muestreos realizados desde 16 días antes de floración (daf) hasta 20 días después de floración (ddf). El PSE se vio afectado por los tratamientos de radiación aplicados 11 daf arrojando diferencias significativas entre el testigo y los tratamientos con sombreo $(\mathrm{p}=0.0023$ y $\mathrm{p}=0.0033$, respectivamente). A 4 daf, las diferencias fueron significativas entre los 3 tratamientos $(p=0.0013)$. El PSE obtenido después de floración (1 y 5 ddf) mostró diferencias entre el testigo y los tratamientos con sombreo, no siendo significativas entre S50 y S80 $(\mathrm{p}=0,0004)$.

El PSE 20 ddf no mostró diferencias significativas entre los tratamientos, esto puede atribuirse a una compensación ocurrida luego que se retiró el sombreo.

Tabla 2. Componentes numéricos del rendimiento: Rendimiento en grano $\left(\mathrm{kg} \mathrm{ha}^{-1}\right)$, peso de 1000 granos (g), Número de granos $\left(\right.$ grano. $\left.^{-2}\right)$ y Número de espigas (espigas. $\mathrm{m}^{-2}$ ) para tres niveles de sombreo. Valores promedios obtenidos de experimentos de campo en diferentes años

\begin{tabular}{|c|c|c|c|c|}
\hline TRATAMIENTO & $\begin{array}{l}\text { RENDIMIENTO } \\
\left(\mathrm{kg} \mathrm{ha}^{-1}\right)\end{array}$ & $\begin{array}{c}\text { PESO }(\mathrm{g}) \\
1000 \text { granos }\end{array}$ & $\mathrm{N}^{\circ}$ DE GRANOS $\mathrm{m}^{-2}$ & ESPIGAS. $m^{-2}$ \\
\hline TESTIGO & $6895.55 \mathrm{c}$ & $40.00 \mathrm{~b}$ & $17245.91 \mathrm{c}$ & $881.00 \mathrm{~b}$ \\
\hline S 50 & $5099.52 \mathrm{~b}$ & $37.63 \mathrm{a}$ & $13430.04 \mathrm{~b}$ & $860.10 \mathrm{~b}$ \\
\hline S 80 & $3402.10 \mathrm{a}$ & $37.90 \mathrm{a}$ & $9267.36 \mathrm{a}$ & $660.25 a$ \\
\hline Fuente de variación & \multicolumn{4}{|c|}{ P valor } \\
\hline TRATAMIENTO & 0.0002 & 0.0930 & 0.0004 & 0.0390 \\
\hline LSD* $^{*}$ & 1112.359 & $1.821^{* *}$ & 2.025 & 179.569 \\
\hline $\mathrm{CV}$ & 13.32 & 3.93 & 13.98 & 14.96 \\
\hline
\end{tabular}

${ }^{*}$ Diferencia limite de significancia al 0.05

** Diferencia limite de significancia al 0.1
Estos componentes numéricos se definen en forma sucesiva a través del ciclo del cultivo y ayudan a explicar, retrospectivamente, en qué etapa y qué factor produjo una reducción en el rendimiento, atribuido al número de granos. Sin embargo, el trigo posee una notable capacidad de compensación entre componentes, esto es, el aumento de uno al disminuir otro, con poco cambio en el resultado final (Abbate et al., 1994), sólo en condiciones potenciales de producción.

Las funciones que predicen el crecimiento de las espigas durante el período crítico fueron ajusta-

Tabla 3. Peso seco de las espigas (PSE) $\left(\mathrm{g} \cdot \mathrm{m}^{-2}\right)$ para diferentes momentos de muestreo durante el período crítico de la variedad de trigo Baguette Premium 13. Valores promedios de dos campañas

\begin{tabular}{lcccccc}
\hline TRATAMIENTO & PSE 16daf $^{* *}$ & PSE 11daf & PSE 4daf & PSE1ddf $^{* * *}$ & PSE5ddf & PSE 20ddf \\
\hline TESTIGO & $6,61 \mathrm{a}$ & $9,04 \mathrm{~b}$ & $38,64 \mathrm{c}$ & $144,16 \mathrm{~b}$ & $145,82 \mathrm{~b}$ & $183,91 \mathrm{a}$ \\
S 50 & $4,07 \mathrm{a}$ & $5,46 \mathrm{a}$ & $27,96 \mathrm{~b}$ & $92,38 \mathrm{a}$ & $81,38 \mathrm{a}$ & $151,45 \mathrm{a}$ \\
S 80 & $4,27 \mathrm{a}$ & $6,29 \mathrm{a}$ & $15,36 \mathrm{a}$ & $80,38 \mathrm{a}$ & $93,52 \mathrm{a}$ & $138,09 \mathrm{a}$ \\
\hline Fuente de variación & \multicolumn{7}{c}{ p valor } \\
\hline TRATAM. & 0,0023 & 0,0338 & 0,0013 & 0,0004 & 0,0004 & 0,1555 \\
DLS* & 1,2605 & 2,6616 & 9,5902 & 23,1844 & 23,50145 & 49,6552 \\
CV & 24,02 & 21,95 & 13,72 & 13,74 & 19,67 & 15,81 \\
\hline
\end{tabular}

* Diferencia limite de significancia al 0,05

** Días antes de floración. *** Días después de floración. 
das según Abbate et al. (1997a) (Fig 1). Para el testigo sin sombreo $\mathrm{y}=6.74+162.27 /(1+\mathrm{e}(-(\mathrm{x}+1.64)) / 1.79))$ $\mathrm{R}^{2}=0,98$; para el tratamiento $\mathrm{S} 50, \mathrm{y}=-8.97+170.62 /$ $(1+\mathrm{e}(-(\mathrm{x}-2.77)) / 6.48)) \mathrm{R}^{2}=0,97$; y para el tratamiento $\mathrm{S} 80, \mathrm{y}=0.70+131.48 /(1+\mathrm{e}(-(\mathrm{x}-0.17)) / 3.064)) \mathrm{R}^{2}=0,95$.

El testigo, en comparación con los tratamientos S50 y S80, presentó el mayor peso de espigas un día después de floración, correspondiéndole el mayor número de granos. El S80 mostró los valores más bajos en peso de espigas un día después de floración y en número de granos. El S50 mostró valores intermedios (Fig 2). El peso de las espigas un día después de la floración tiene una relación lineal positiva con el número de granos.

Según Abbate et al (1998) cuanto mayor es el peso seco de las espigas, mayor es la disponibilidad de fotosintatos, y mayor el número de granos por unidad de superficie. El número de granos por $\mathrm{m}^{2}$, está relacionado con el peso seco que alcanzan las espigas (excluido el peso del grano) siete días después de ocurrida la floración; por lo tanto mientras más propicias sean las condiciones para el crecimiento de las espigas, se puede esperar un mayor número de granos por unidad de superficie. Esto se logra si durante el período de crecimiento de las espigas se intercepta la mayor cantidad de radiación posible (Cantarero et al., 1998). Debido a que el tallo y la espiga crecen en forma coetánea en el tiempo durante la última etapa de la encañazón, la competencia por recursos entre ambos órganos es un aspecto crucial para el crecimiento de la espiga, ya que es el órgano de mayor importancia en término de rendimientos. Durante esta etapa, una mayor partición de asimilados hacia las espigas, se traducirá en un mayor número de granos al momento de la cosecha (Miralles, 2003).

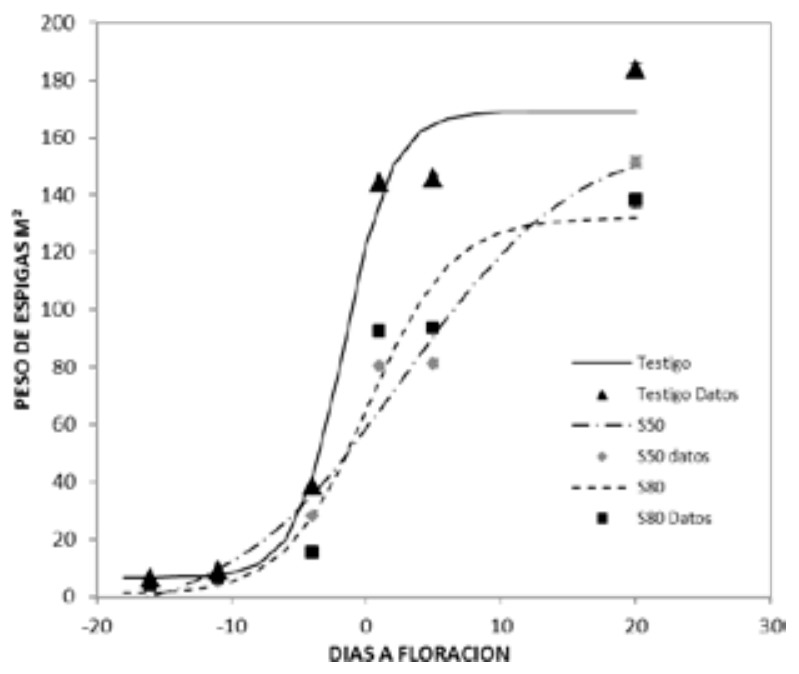

Fig 1: Relación entre el PSE. $\mathrm{m}^{-2}$ y los días a floración durante el periodo crítico. Las líneas corresponden a la ecuación ajustada a los datos experimentales para cada tratamiento
Tabla 4: Tasa de crecimiento diaria de espigas y eficiencia del uso de la radiación de las espigas para los tres tratamientos con diferentes niveles de sombreo durante el período crítico.

\begin{tabular}{lcc}
\hline TRATAMIENTO & $\begin{array}{c}\text { TCE } \\
\left(\mathrm{g} \cdot \mathrm{m}^{-2} \cdot \mathrm{dia}^{-1}\right)\end{array}$ & $\begin{array}{c}\text { EUR de espigas } \\
\left(\mathrm{g} \cdot \mathrm{m}^{-2} \mathrm{dia}^{-1} \mathrm{MJ}^{-1}\right)\end{array}$ \\
\hline TESTIGO & 10,3940231 & 1,9300659 \\
S 50 & 5,76238462 & 1,99065307 \\
S 80 & 6,62291731 & 4,10829876 \\
\hline
\end{tabular}

Según varios autores (Abbate et al., 1994 y 2004; Cantarero et al., 1998; Miralles, 2003) si las particiones a espigas son similares durante el periodo crítico existe una alta relación entre el peso seco de las espigas y el número de granos. En este trabajo se encontró una asociación $\mathrm{r}=0.62$, esta relación podría tener más consistencia si las particiones a espiga hubieran sido iguales, sin embargo existieron diferencias aunque no fueron significativas.

Se analizó la tasa de crecimiento de espigas desde 10 días antes de floración hasta el día de floración (momento en que la curva de crecimiento se hace exponencial Fig 1), siendo para el testigo la TCE diaria $10.39 \mathrm{~g} \mathrm{~m}^{-2} \mathrm{día}^{-1}$, para el tratamiento $\mathrm{S} 50$ de $5.76 \mathrm{~g}$ $\mathrm{m}^{-2}$ día $^{-1}$ y para el tratamiento $\mathrm{S} 80$ de $6.62 \mathrm{~g} \mathrm{~m}^{-2}$ día $^{-1}$. Luego se analizó la EUR de espigas, arrojando los siguientes resultados: testigo $1.93 \mathrm{~g} \mathrm{~m}^{2} \mathrm{dia}^{-1} \mathrm{MJ}^{-1}$, S50: $1.99 \mathrm{~g} \mathrm{~m}^{2}$ día $^{-1} \mathrm{MJ}^{-1}$ y S80: 4.11 gm $^{2}$ día $^{-1} \mathrm{MJ}^{-1}$ (Tabla 4). De estos datos surge que en el tratamiento S80 hubo mayor TCE diaria por cada MJ acumulado durante ese período, logrando mayor eficiencia que el tratamiento S50, sin embargo esto no se tradujo a un mayor peso de espigas ni a un mayor número de granos.

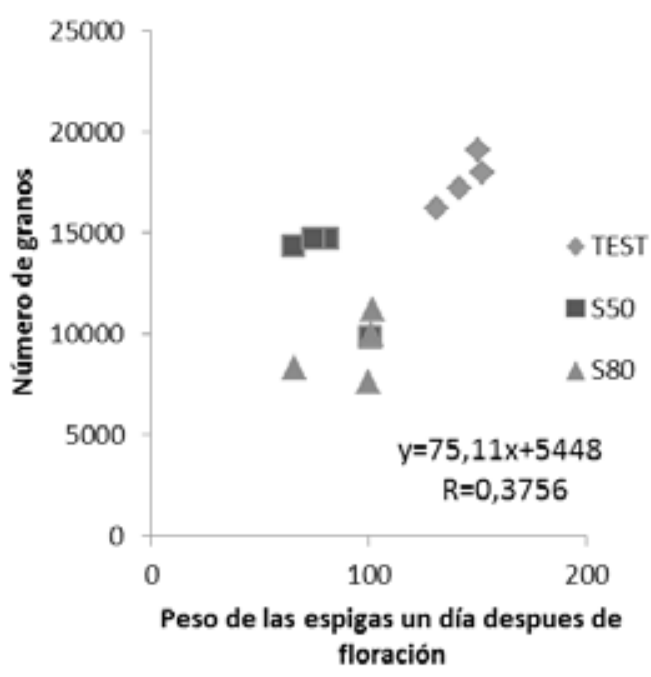

Fig 2: Relación entre el número de granos por metro cuadrado y el peso de las espigas un día después de floración para los diferentes tratamientos. 


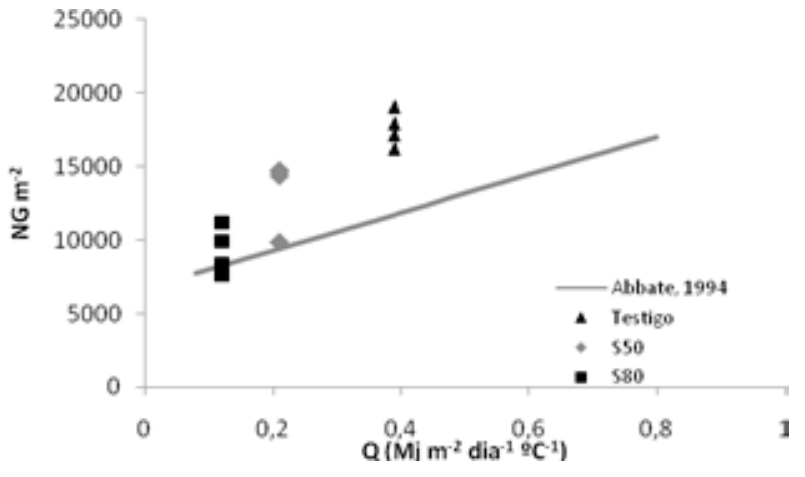

Fig 3: Relación entre el número de granos $/ \mathrm{m}^{2}$ y el cociente fototermal (Q) durante el periodo crítico de la variedad de trigo Baguette Premium 13. La línea continua representa la ecuación propuesta por Abbate et al., (1994). Los símbolos representan los experimentos de campo para distintos tratamientos.

En la Fig 3 se observa la relación teórica propuesta por Abbate et al., (1994) entre el número de granos potencial y el cociente fototermal durante el período crítico calculado mediante el cociente entre la RFA y la temperatura media menos $4.5^{\circ} \mathrm{C}$. La recta representa la ecuación: $\mathrm{NG}=6763+9815 \mathrm{Q}(\mathrm{r}=0.88 ; \mathrm{gl}=18)$. (Abbate et al., 1994). Los datos de este trabajo se representaron en la misma figura, el testigo (triángulos verdes), el sombreo 50 (cuadros azules) y el sombreo 80 (cuadros rojos); los cuales se ubicaron siempre por encima de la recta teórica. Esto puede deberse a que los datos de este ensayo se obtuvieron con material genético de una década posterior a la cita y con incorporación de germoplasma de trigos franceses (Abbate et al., 1994).

Con la radiación y temperaturas registradas durante el periodo crítico promedio de ambas campañas se obtuvo un valor de Q para el testigo de $0.405 \mathrm{MJ}$ $\mathrm{m}^{-2} \mathrm{dí}^{-1}{ }^{\circ} \mathrm{C}^{-1}$, en el cual el número de granos por metro cuadrado fue 17245.91. Para los tratamientos con sombreo el $\mathrm{Q}$ fue menor a medida que se aumentó el nivel de sombreo, $0.21 \mathrm{MJ} \mathrm{m}^{-2}$ día $^{-1}{ }^{\circ} \mathrm{C}^{-1}$ para S50 y $0.12 \mathrm{MJ} \mathrm{m}^{-2}$ día $^{-1}{ }^{\circ} \mathrm{C}^{-1}$ para $\mathrm{S} 80$, y así disminuyó el número de granos, 13430.04 y 9267.36 para S50 y S80, respectivamente (Fig 3).

\section{CONCLUSIONES}

El trigo variedad Baguette Premium 13 con fecha de siembra óptima conducido en condiciones potenciales, tuvo un rendimiento de más de $6800 \mathrm{~kg} \mathrm{ha}^{-1}$. Cuando se disminuyó la radiación incidente mediante sombreos, éste disminuyó a 5099 y $3402 \mathrm{~kg} \mathrm{ha}^{-1}$ para disminuciones del 46 (S50) y 70\% (S80) de la radiación incidente, respectivamente.

El rendimiento final fue explicado en un $98 \%$, tomando en cuenta todos los niveles de radiación incidente, por el numero de granos; y éste último se asoció positivamente $(\mathrm{r}=0.62)$ con el peso de espigas al finalizar el período crítico.

La tasa de crecimiento de espigas durante el período crítico fue explicada por tres ecuaciones, una para cada nivel de radiación; en las parcelas con bajos niveles de radiación, la eficiencia de su uso por parte de las espigas fue mayor que en el testigo aunque no se llegan a compensar los pesos de espigas.

\section{BIBLIOGRAFIA}

Abbate, P.; Andrade, F. y Culot, J. 1994. Boletín Técnico $\mathrm{N}^{\circ} 133$. EEA INTA Balcarce. $17 \mathrm{pp}$.

Abbate, P.; Andrade, F. y Lázaro, L. 1997 (a). Explorando altos rendimientos de trigo. CIMMYT-INIA. 20 pp.

Abbate, P.E.; Andrade, F.H. y Culot,J.P. 1997 (b). Grain yield in wheat: Effects or radiation during spike growth period. Filed crops Research 54,245-257.

Abbate, P. 1998. Ecofisiología del cultivo de trigo. Conferencia Congreso Nacional AAPRESID. Balcarce, Bs. As. 6 pp.

Abbate, P. 2004. $12^{\circ}$ Conferencia Congreso Nacional de AAPRESID. 12 de agosto. Rosario, Sta. Fe. 9 pp.

Bruniard, E. 2000. Los regímenes climáticos y la vegetación natural. Aportes para un modelo fitoclimático mundial. Academia Nacional de Geografía. Publicación Especial No 16. Bs. As., Argentina. 79 pp.

Cantarero, M.; Dardanelli, J. y Badiali, O. 1998. Factores ambientales que determinan el rendimiento potencial en trigo. Boletín INTA. Balcarce, Bs. As., 9 pp.

De Fina, A.L. y Ravelo, A.C. 1985. Climatología y Fenología Agrícolas. $4^{\circ}$ Ed. EUDEBA.Bs. As., Argentina. 354 pp.

Escobar, E.H.; Ligier, D.; Melgar, M.; Matteio, H. y Vallejos, O. 1994. Mapa de suelos de los Departamentos de Capital, San Cosme e Itatí de la Provincia de Corrientes, Argentina. Publicación del Convenio del Instituto Nacional de Tecnología Agropecuaria (INTA-ICA) y Provincia de Corrientes-CFI, Argentina. $129 \mathrm{pp}$.

Fischer, R.A. 1985. Number of kernels in wheat crops and the influence of solar radiation and temperature J.Agric. Sci., Camb, 105, 447-461.

Gallo, W.P., y C.S.T. Daughtry. 1986. Techniques for measuring intercepted and absorbed photosynthetically active radiation in crop canopies. Agron. J. 78:752:-756.

Miralles, D.J. 2003. Fisiología de la generación del rendimiento. http://www.eemac.edu.uy/dmdocuments/ cereales_y_cultivos_industriales_A/cultivos_de_invierno/fisiologia_del_rendimiento/Miralles-fiologia del_rendimiento-Trigo.pdf

Pascale, A.J. y Damario, E.A. 2004. Bioclimatología Agrícola y Agroclimatología. Editorial Facultad de Agronomía, Universidad de Buenos Aires.550 pp. 
Satorre, E.H.; Benech A., R.; Slafer, G.A.; De la Fuente, E.B.; Miralles, D.J.; Otegui, M.E. y Savin, R. 2003. Producción de Granos: Bases Funcionales para su Manejo. Editorial Universidad de Buenos Aires. 576 pp.

Seghezzo, M.L. y Molfese, E.R. 2006. Calidad en trigo pan. Boletín técnico $N^{\circ} 41$. INTA. Chacra Experimental Integrada Barrow- Buenos Aires. Ediciones INTA. 35 pp.
Slafer G. A., Andrade F. H. and Satorre E. H. 1990.Genetic improvement effects on pre-antesis physiological attributes related to wheat grain yield. Field Crops Research 23, 255 - 263.

Strahler, A.N. y Strahler, A.H. 1997. Geografía Física. $3^{\circ}$ Edición. Ed. OMEGA. Barcelona, España. 550 pp. 